\title{
A simple and generic interface for a cloud monitoring service
}

\author{
Augusto Ciuffoletti \\ Dipartimento di Informatica - Università di Pisa \\ augusto@di.unipi.it
}

Keywords: Cloud Monitoring, Open Cloud Computing Interface (OCCI), RESTful service, Cloud Ontology, Cloud Interoperability

\begin{abstract}
The paper addresses the definition of an ontology for cloud monitoring activities, with the aim of defining a standard interface for their configuration. To be widely adopted, such ontology must be extremely flexible, coping with a wide range of use cases: from the minimalist plug-and-play user, to the one governing a complex infrastructure.

Our work is based on the Open Cloud Computing Interface, that is an open, community driven OGF standard allowing boundary-level interfaces to be built using RESTful patterns over HTTP. Among others, OpenStack and OpenNebula adopt OCCI.

Using the OCCI ontology we define two kinds that are associated with the basic components of a monitoring infrastructure: the collector link, that performs measurements, and the sensor resource, that aggregates data and undertakes actions.

This paper is a compact and self-contained revision of a document currently under discussion inside the OCCI community.
\end{abstract}

\section{Introduction}

In the current terminology a Cloud is a collection of resources that are dynamically provisioned to a user: see for instance the NIST definition in (Mell and Grance, 2011). Whenever the task such resources are involved in becomes critical, for any of the many reasons and ways a computing task may become critical, the user wants to evaluate its performance: the NIST definition referenced above is explicit about this fact, and indeed a growing attention is paid to the topic of cloud monitoring.

The purpose of cloud monitoring is to extract quantitative performance evaluations of cloud resources in the form of metrics. During the operation, cloud monitoring produces measurements, that are instances of given metrics.

The measurements produced during monitoring are used for several reasons. Among them:

- Fault Treatment - historically this is the main purpose for resource monitoring. However a Cloud Computing environment gives a different perspective, since hardware failures now are softly coped with by the cloud provider (VMware, 2007). Fault treatment is still needed for marginal conditions that damage the application while be- ing admitted by the Service Level Agreement (SLA) stipulated with the provider;

- Billing - the provider expects a revenue that is related with the amount of resources consumed by the user. A billing rule that is adherent to the effective use, and not to the reserved resources, is more effective from both the user and the provider side: the user pays only for what it uses, and the provider has further optimization margins;

- Service Level Agreement verification - the user that signs a service level agreement with the provider wants to be able to verify that the service meets the terms of the contract. The provider that meets this demand increases its credibility;

- Quality of Service implementation - the user that implements a service in the cloud may want to ensure a given QoS whose terms are different from the SLA signed with the cloud provider: the availability of resource performance metrics enables the user to undertake actions to ensure the QoS.

The above non-exhaustive taxonomy justifies the claim that the monitoring activity deeply depends on user needs: the provider is in charge of making available the resource measurement tools, but the user is left the final word about their configuration to meet 
specific use cases.

This paper aims at the definition of an ontology for the domain of cloud monitoring, thus defining the contents crossing the interface between the provider and the user, in view of the definition of a widely accepted standard.

In the next section we give a taxonomy of use cases, and then we explore the monitoring tools currently offered by a selection of cloud providers.

\section{An ontology for cloud monitoring}

The monitoring of cloud resources is a complex task, and many entities contribute to its unfolding. It is therefore appropriate to identify a limited set of fundamental concepts - an ontology - that helps the coordination among the involved parties. This approach has been already adopted in cloud computing (Youseff et al., 2008; Di Modica et al., 2012; Bernstein and Vij, 2010), and it is tightly related with interoperability and service discovery.

We first observe that the final user of measurements does not always identify with the user of the monitored infrastructure. We distinguish three alternatives:

- the user of resource measurements is the cloud provider. In this case monitoring is directed either to billing purposes using raw usage metrics on real hardware resources, or to implement a Service Level Agreement,

- the user of resource measurements is the cloud service end-user. In this case the user is mostly interested in checking Service Level Agreement Compliance, or in the implementation of application-specific resource optimization,

- the user of resource measurements is a user that, in his turn, provides critical services based on resources leased by another provider. The case is similar to the above, but with a number of challenging variants since the measurements may come from dynamically provisioned virtual resources from both a private and public clouds.

The way raw measurements are processed before being delivered introduces further variance in the scenario: resource performance is usually expressed as a multi-dimensional metric, while an aggregate unidimensional metric, tailored for a specific application, is more appealing for the user. For business related reasons, also the provider prefers to expose an aggregated metric, instead of a fine grain set of raw metrics.

An example is the measurement of network performance in an IaaS cloud, since performance mea- surements should be dynamically configured on demand, depending on a changing user activity: the provider has to figure out the filters to be applied to networking devices so to select and measure relevant traffic characteristics.

The description of resource monitoring capabilities becomes relevant when it is considered as a part of the provided service, considering the monitoring infrastructure itself as a leased resource. Its description should cross the same interface used to convey the description of the service.

There are currently a lot of such interfaces, which is one of the problems a potential cloud user finds on his way. Cloud providers want to make their services more attractive, and thus they design straightforward interfaces for their specific provision. Such an approach tends to fragment the access to cloud services into a number of narrow, vendor-specific interfaces: let us explore the offer.

\subsection{Cloud monitoring in the market}

The majority of cloud providers offer resource monitoring as part of the provided service: at a point in time, the user may decide to subscribe to an additional service that reports measurements or that undertakes actions based on the monitoring activity.

The AWS monitoring service is named CloudWatch. It is available for all of the AWS resources, like compute resources, various forms of database and storage facilities, and other infrastructure components like queues and load balancers. In total, more than one hundred metrics are available. The typical frequency is of one measurement every 5 minutes, but periods as low as 1 minute are allowed. One notable fact is that, among the available metrics, there is the financial cost of the cloud provision. The measurements may be directly used to trigger compensating actions in a closed control loop, or they may be presented for human inspection. To improve flexibility, AWS allows the user to perform custom measurements, and to submit them as an input to CloudWatch, for instance to shut down an unused resource.

AzurWatch solution has a typical period of one hour, and around twenty metrics are offered on the various components of a cloud provisioning, that are either used as a trigger for elastic behaviors, or directly presented.

RackSpace approach consists of providing a software package that implements a Monitoring Agent. Once installed on a compute instance in the cloud, it is able of producing measurements from that server. The user controls which measurements are collected and how they are used through a management page, 
which is embedded in the cloud management interface. In addition, measurements feed automatic actuators represented with checks: for instance, one check consists in sending a series of ping to an IP address and reporting problems by email.

Private cloud platforms may live without a structured way to deal with monitoring: for instance, the open cloud computing platform OpenStack does not provide a structured solution, but its open source nature lets the user free to implement a custom solution.

In contrast, OpenNebula offers both a nuts and bots solution based on software probes that push measurements to the cloud monitoring front-end, and a closed loop mechanism, that implements automatic scaling decisions based on measurements. OpenNebula offers also a less scalable pull mechanism based on the same software probes.

A totally different approach consists of the provision of a stand-alone monitoring service. For instance, StackDriver provides an agent that is installed on the user premises to monitor cloud resources: it is designed to inter-operate with the monitoring services offered by AWS and RackSpace clouds. The Stackdriver dashboard integrates the know-how that helps the user to design an effective analysis of the measurements, thus obtaining sensitive and reliable alarms.

CompatibleOne is a project that follows a similar approach, and aims at providing the widest possible compatibility with existing cloud computing platforms: a unique tool to interact with all major providers. Therefore they embed in an interface the tools to describe a monitoring framework, that envisions the presence of a specialized monitoring agent coordinating the monitoring probes activity.

After this partial overview, we understand that there are a lot of ways to provide a cloud monitoring service, which is regarded as a positive symptom of vitality, and we expect new and innovative ways to emerge. An attempt to contain them within a rigid standard would be a mistake, ultimately unsuccessful. On the other hand, the existence of an agreement about a few relevant aspects allows interoperability, with all of the advantages that we learned from the history of the Internet.

The challenge is in finding the balance between what is specified in the standard, and what is left unspecified, so that a new service can be both innovative and complying.

\subsection{The OCCI way to cloud standards}

The OCCI approach to the definition of a standard for cloud computing (Edmons et al., 2012) consists in defining the interface to the service, giving as little detail as possible to the definition of the service itself. The interface is defined in a way that is easily implemented with tools that, in their turn, are firmly based on widely used and accepted standards.

The document that describes the core of the OCCI standard (OGF, 2011a) lays the foundations, binding the cloud management interface to be RESTful, according with the principles defined in (Fielding and Taylor, 2002). This gives an HTTP framework to the interface between the cloud user and the provider, and restricts the interaction between the two so to preserve the advantages of the HTTP infrastructure (like proxying, caching etc.). According to the REST paradigm, the resource with its representation are subject of the communication between a client and the server.

Besides the core standard, designed to be longliving as valuable standards need to be, there is a series of satellite documents, that enrich the core with the details of a specific interface and provisioning. One series of standards specifies the language used to represent entities, i.e. its rendering. At this time, there are three such renderings: embedded as HTTP attributes, using the JSON language and, the last to appear and not yet fully specified, using XML. Another series of standards is bound to a specific kind of cloud provisioning: at this time there is one such document that focuses on an IaaS provisioning, defining entities like compute and storage resources.

The proposal addressed in this document is in the spirit of defining resource monitoring as just another kind of cloud service provisioning ${ }^{1}$. Since we use the OCCI ontology, we summarize the features of the OCCI API scheme before going into the details of our proposal.

\section{OCCI - An open cloud computing interface}

The OCCI working group of the Open Grid Forum (OGF) has produced the description of an interface for the description of a Cloud Computing infrastructure. It representes the front gate of a cloud provider: the users willing to obtain resources from a provider need to submit a request that follows a given protocol. The intent of the OCCI working group is to foster the convergence towards a widely adopted standard.

Both users and providers have an interest in the existence of a standard, since its introduction usually

\footnotetext{
${ }^{1}$ The concepts exposed in this paper are the basis of a more technical paper, that can be found browsing the repository of the OCCI project at http://redmine.ogf.org, in git branch named monitoring
} 
carries a development in the market as well as technology advances. The ideal place for its development is not a private enterprise, since it is subject to pressures from the market. A better result is expected from an independent forum, where the suggestions coming from industries may meet with the results of independent research: the OGF, together with other standardization bodies, played an important role on this respect, and the OCCI working group is a branch of it.

The OCCI interface is based on a server that operates following the Representational State Tranfer (REST) paradigm. According with it, the client and server exchange messages that contain the description of resources: in this context, the resource is any concept that admits a formal representation, and its is not directly related with a computing resource, whose monitoring is the matter of our investigation.

In a REST framework (Fielding and Taylor, 2002), the client and the server exchange request and response messages that contain representations of the state of REST resources. In this respect the REST framework defends a coherent utilization of the HTTP protocol, against new communication tools like WebSockets, by introducing four fundamentals constraints:

- the communication between the client and the server uses a uniform interface carrying the representation of resources

- there is no session-related state in the server, and each request-response pair is a self-contained operation

- the existence of an intermediate processing of the messages (like caching) is trasparent

- the client may provide code to extend server capabilities

The request message contains the indication of an operation to be performed on the resource: the REST paradigm indicates four operations that correspond to the well known HTTP verbs: GET, PUT, POST and DELETE.

The OCCI interface uses a REST interface to describe the interaction between the user and the service provider aimed at the specification of the infrastructure the user wants to obtain from the server. A great deal of attention is paid to the structure of the information that may be included in the message, and that describes the operation of infrastructure resources.

According with OCCI proposal, the representation of a cloud infrastructure is carried out by describing REST resources represented as instances of an entity type. An entity instance is characterized by a unique identifier, but it is otherwise left abstract: it needs to be related with a kind and to one or more mixins in order to be fully specified. The kind gives an entity its basic features, described as attributes. Kinds are arranged in a tree structure, where each kind is put into relationship with another higher level one, in a sub-typing hierarchy.

The OCCI working group has defined two core kinds: the resource, here intended as an IT resource ${ }^{2}$, and the link, that represents a relationship between $r e$ sources. Each core type can be sub-typed in its turn to take into account the multitude of IT resources and their relationships, thus generating the kinds hierarchy.

The association of a mixin to an entity instance corresponds to a further characterization of it. A mixin can be used to bind attributes already defined by the kind, or to introduce new attributes, like a root filesystem with a preconfigured OS. Mixins are related with a many-to-many relationship: in particolar, a mixin can be defined as a tag with an associated semantic, but no attributes. The UML class diagram that describes the OCCI model is in figure 1, and its exhaustive definition is in (OGF, 2011a).

The core ontology is in fact more general than what strictly needed for the definition of an IaaS cloud. In a distinct document (OGF, 2011b) the OCCI working group defines a specialization of the core model that addresses such task. In a nutshell, three sub-kinds of the resource kind are defined that model IaaS resources (Compute, Network and Storage), and two sub-kinds of the Link kind to describe relationships among them (NetworkInterface and StorageLink).

In the end, the task of describing a cloud infrastructure is carried out in a natural with the instantiation of a number of Compute, Storage and Network resources, configuring them with appropriate attribute values, and interconnecting them using StorageLinks and NetworkInterfaces links. Appropriate mixins are associated to those entities that need further specification.

Note that the hierarchical nature of kinds and mixins allows the user to discover the capabilities of a provider: for instance, the availability of a Celeron CPU might be discovered browsing the mixins that can be associated with a Compute entity. In fact, a provider may define provider-specific mixins, thus leaving the overall structure open to unlimited extension.

\footnotetext{
${ }^{2}$ in this document the term resource is used in two quite different but close meanings: one is the content addressed with an URI, as defined in sec. 1.1 of (Berners-Lee et al., 2005), the other is the representation of a cloud resource. To disambiguate we use the term REST resource for the former
} 


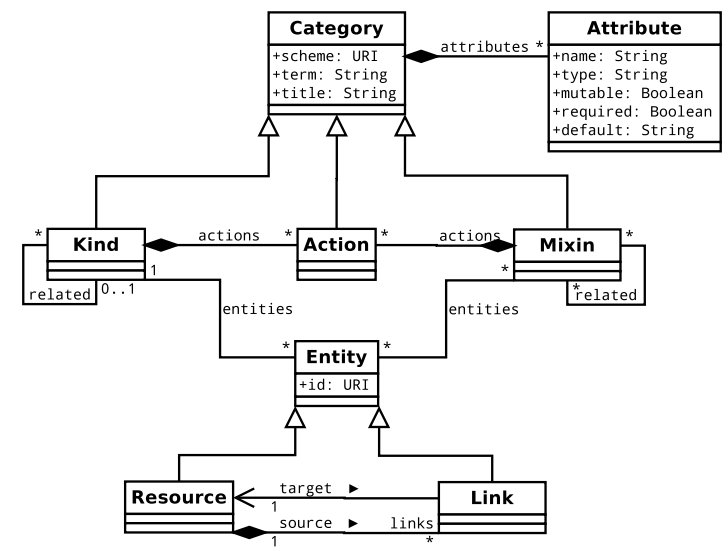

Figure 1: The OCCI core model - A UML class diagram Copyright (c) Open Grid Forum (2009-2011). All Rights Reserved

All operations involved in the instantiation of Entities in the above scheme are carried out using the basic HTTP verbs: once a system is populated with Entities, it can be browsed using associations. For instance, the discovery of available resources is implemented with a GET / /, while the instantiation of new entities is obtained with a POST. The descriptions of the available mixins is learned using the above discovery mechanism, and mixin instances are associated to Entity instances using POST requests.

The next step is to introduce an OCCI extension to describe a monitoring infrastructure.

\section{Definition of a monitoring infrastructure using OCCI}

In our view, a monitoring infrastructure can be defined as a service using an extension of the OCCI core model: this approach follows the one used to define an IaaS in the OGF document (OGF, 2011b). The adherence to the OCCI model is deemed appropriate, since that standard has the favorable properties of being open, and of being designed to be long-living. There is no need to introduce a dependency from the IaaS entities defined in (OGF, 2011b), and so our monitoring framework may extend to other computational models, different from a plain IaaS.

In a simplistic view, a monitoring capability might be associated to a generic resource using a mixin describing the available metrics, in analogy with the OpenNebula pull mechanism. However this scheme is able to capture only very simple cases, and it is unable to model a metric that aggregates several raw metrics, like the average load of a pool of servers. In addition, the inclusion of measurements in the render- ing of a resource introduces scalability issues, since such attributes are frequently updated, and devoids caching: it is definitely in contrast with the RESTful paradigm.

Instead, our option is to introduce a specialized resource kind, that we call sensor, that embeds the capabilities of processing and delivering the measurements: it is not directly involved with their production. It can be approached to the monitoring agent as introduced by CompatibleOne and Stackdriver, and that we successfully adopted in a Grid-oriented research prototype (Ciuffoletti et al., 2003; Ciuffoletti and Polychronakis, 2007). A single sensor is able to collect measurements from many sources, and to deliver metrics obtained processing the inputs in many ways.

The user defines across the interface the timing of the sensor resource, and operational details are defined using provider specific mixins: the definition of the available monitoring capabilities is thus left to the provider. This is appropriate since the provider wants to define monitoring capabilities according with its own business strategy: as shown in the introductory overview, each provider has a distinguished one.

We consider that a representative ontology must be powerful for the demanding user, and simple for minimal tasks: on one end we want a simple metric (in the values $\{$ green, yellow, red $\}$ ) for a large infrastructure moving the complexity in the aggregation of raw metrics (in Rackdriver style), on the other we want a raw metric on a single resource $(\mathrm{Mb} / \mathrm{s}$ on a network interface) (in RackSpace style). A truly generic ontology must capture both use cases, as well as all those in between. So we define a designated resource whose task is to represent and coordinate the monitoring activity.

The next step is the description of the relationships between the sensor and the resources from which it receives measurements: it is a way to associate a metric to a (resource,sensor) pair. The link entity is the tool offered by the OCCI core model for this case. The metric is thus attached to a link between a sensor and a generic resource: we call collector the kind of this link. This solution has the favorable property of making discoverable the association between sensors and monitored resources. It can be approached with Rackspace and CompatibleOne probe.

Like in the case of the sensor, the generic interface transfers only the timing aspects of the collector, and leaves to provider-specific mixins the work of giving the operational definition of the collector.

The introduction of the sensor and of the collector subtypes can be described in the UML diagram that is shown in figure 2. 


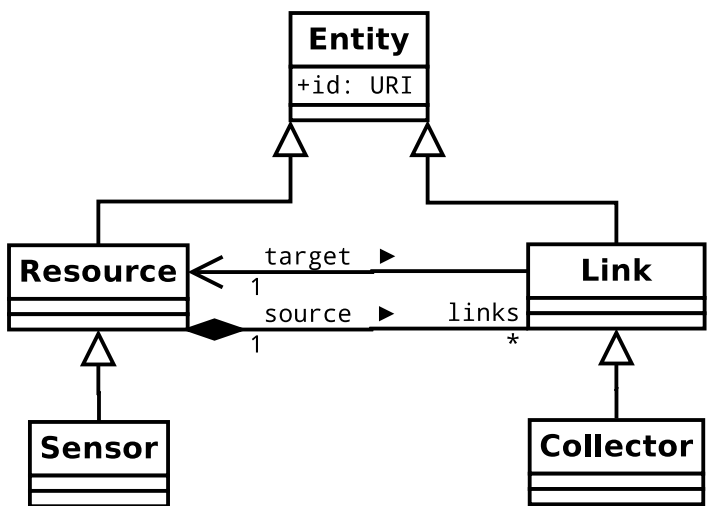

Figure 2: A model for monitoring entities - UML class diagram

In conclusion, to introduce a monitoring service in an existing infrastructure we proceed as follows:

1. we attach an instance of a collector link to each of the monitored resources, and define the collected metrics by way of mixins;

2. we define a sensor that receives the measurements from the collectors and associate processing and publishing funcionalities by way of mixins;

The approach defined above does not allow a plain pull mechanism, consisting in a direct access of metric attributes inside the resource description. Such option has an heavy footprint on the provider, and it is not RESTful compliant: so we consider this restriction as justified.

With some effort, it is possible to treat very simple use cases by unifying the monitored resource and the sensor, so that the two functionalities live together inside the same resouce: this is near to the RackSpace solution. However, the same solution can be represented in a cleaner way using two distinct entities.

\section{Description of REST resources for cloud monitoring}

This section is devoted to the description of the two abstract REST resources in our ontology: the sensor resource and the collector link.

A sensor resource defines the timing of the measurements (see table 1): how frequently measurements are collected, during which time period, the accuracy and the granularity of the time scale. Any further specification is left to mixins that describe the relevant aspects of the sensor, namely:

- the way input metrics are aggregated to produce an output metric and

\begin{tabular}{|c|c|c|c|c|}
\hline $\begin{array}{l}\text { Model } \\
\text { attribute }\end{array}$ & value & & & \\
\hline \multirow{4}{*}{$\begin{array}{l}\text { scheme } \\
\text { term } \\
\text { title }\end{array}$} & \multicolumn{4}{|c|}{ http://schemas.ogf.org/occi/monitoring\# } \\
\hline & \multicolumn{4}{|l|}{ sensor } \\
\hline & \multicolumn{4}{|l|}{ Sensor Resource } \\
\hline & name & type & mut. & req. \\
\hline \multirow{6}{*}{ attributes } & occi.sensor.period & number & true & true \\
\hline & occi.sensor.periodspec & string & true & false \\
\hline & occi.sensor.timebase & number & false & true \\
\hline & occi.sensor.timestart & number & true & true \\
\hline & occi.sensor.timestop & number & true & true \\
\hline & occi.sensor.timespec & string & true & false \\
\hline
\end{tabular}

Table 1: Definition of the Sensor Resource Kind

- how output metrics are published

To clarify the semantics associated to the two types of mixin, we describe the role of their attributes.

In the case of the mixins that define the aggregation function, we have input attributes that bind the measurements provided by one of the ingress collectors to variables in the aggregation algorithm, and output attributes that bind the results of the aggregation to a mixin that defines the way they are published. Other attributes indicate the value to assign to constant parameters used in the aggregation algorithm.

In the case of mixins that describe a publishing method, we have input attributes that bind the metrics computed locally or coming from ingress collectors to items in the communication protocol. The binding between input and output attributes is implemented with labels, that are assigned as values to input and output attributes. The scope of such labels is limited to the sensor and to the set of input collectors. Example of publishing mixins are those that deliver the measurements through a graphical interface, but also those that store the data in a database, or that trigger cloud management activities in a closed loop scheme.

\begin{tabular}{|c|c|c|c|c|}
\hline $\begin{array}{l}\text { Model } \\
\text { attribute }\end{array}$ & value & & & \\
\hline \multirow{4}{*}{$\begin{array}{l}\text { scheme } \\
\text { term } \\
\text { title }\end{array}$} & \multicolumn{4}{|c|}{ http://schemas.ogf.org/occi/monitoring\# } \\
\hline & \multicolumn{4}{|c|}{ collector } \\
\hline & \multicolumn{4}{|l|}{ Collector Link } \\
\hline & name & type & mut. & req. \\
\hline attributes & $\begin{array}{l}\text { occi.collector.period } \\
\text { occi.collector.periodspec }\end{array}$ & $\begin{array}{l}\text { number } \\
\text { string }\end{array}$ & $\begin{array}{l}\text { true } \\
\text { true }\end{array}$ & $\begin{array}{l}\text { true } \\
\text { false }\end{array}$ \\
\hline
\end{tabular}

Table 2: Definition of the Collector Link Kind

A collector link (see table 2) defines a relationship between a sensor resource and another generic cloud resource. Like the sensor resource, also the collector link has attributes that define the timing of the measurement activity. The operation of a collector instance is further defined by mixins that indicate the collected metrics. Such mixins are characterized by a title that is reminiscent of the measured metric and of 


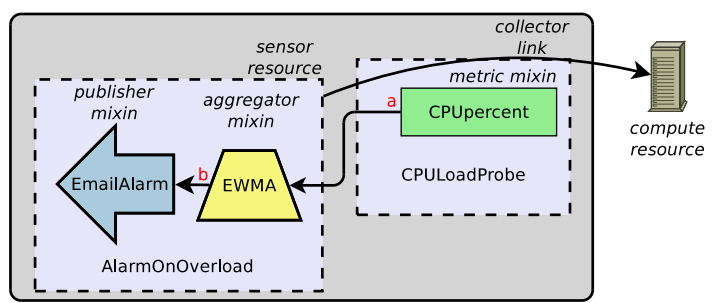

Figure 3: Block representation of a CPU overload alarm

the methodology used to perform the measurement. Other parameters control the application of the specific methodology (e.g, the length of a ping packet), and output attributes convey the measurements to the sensor.

\begin{tabular}{ll}
\hline $\begin{array}{l}\text { Model } \\
\text { attribute }\end{array}$ & value \\
\hline $\begin{array}{l}\text { scheme } \\
\text { term }\end{array}$ & http://schemas.ogf.org/occi/monitoring/collector\# \\
\hline scheme & metric \\
term & http://schemas.ogf.org/occi/monitoring/sensor\# \\
\hline scheme & aggregator \\
term & publisher \\
\hline
\end{tabular}

Table 3: The Mixin tags defined for the monitoring API

As seen, mixins for resource monitoring fall into three categories: those that describe how metrics are aggregated, those that indicate how they are published and those that stand for the metrics themselves. To give a representation of this fact, we introduce three tagging mixins that are used to apply structural constraints that are specific for each set of mixins. The three tags are defined in figure 3 .

These mixins play a fundamental role, since their semantics define the monitoring that is introduced in the system. To leave the provider free to define a business strategy for the monitoring service, they are left defined as not fully qualified entities. In this way the applicability of the proposed ontology is not limited to the compute/storage/network triad: for instance, the scheme described above can be easely adapted to a PaaS infrastructure, where the monitored resource is a NoSQL repository and the metric is the number of queries per second.

Finally, we see how to represent a simple monitoring activity that consists of an alarm that sends an email when the load of a CPU is steadily above a threshold, using a filter to avoid false alarms.

\section{A simple case: alarm for an overloaded CPU}

The user wants to create an alarm that sends an email to a given address when the load of a compute resource (id=urn:uuid:1111) is steadily over $80 \%$. The check is run evey minute starting ten minutes from now, and it lasts during one hour.

The block diagram of the alarm is shown in figure 3 , and we now explain how the user can incrementally implement it using the API defined in this paper.

The first step in the workflow is the instantiation of a blank sensor with the following attributes (optional attributes are omitted):

\begin{tabular}{|l|l|}
\hline name & value \\
\hline title & "AlarmOnOverload" \\
period & "60" \\
timebase & "1386925386" \\
timestart & "600" \\
timestop & "3600" \\
\hline \multicolumn{2}{|l|}{ Alarm0nOverload sensor attributes }
\end{tabular}

The server returns an id=urn: uuid:2222 for the sensor. The next step is the instantiation of the collector link, with timing attributes consistent with those of the sensor:

\begin{tabular}{|l|l|}
\hline name & value \\
\hline title & "CPULoadProbe" \\
source & urn: uuid: 2222 \\
target & urn: :uid: 1111 \\
period & "60" \\
\hline \multicolumn{2}{|l|}{ CPULoadProbe collector attributes }
\end{tabular}

Now the user agent browses the capabilities of the provider looking for one that measures the CPU load, and finds the CPUpercent one. The mixin is added to the CPULoadProbe with a POST carrying the added attribute:

\begin{tabular}{|l|l|}
\hline name & value \\
\hline out & a \\
\hline \multicolumn{2}{l}{ CPUpercent metric attributes } \\
\hline
\end{tabular}

indicating a label attached to the output stream.

Next the user explores provider's capabilities looking for a robust average, an it finds the exponentially weighted moving average inplemented by the EWMA mixin: three attributes are indicated, respectively for the gain, the input and the output stream. The mixin is added to the AlarmOnOverload sensor with a POST and the attributes are given a value: 


\begin{tabular}{l|l|}
\begin{tabular}{l|l|}
\hline name & value \\
\hline gain & "16" \\
instream & $\mathrm{a}$ \\
outstream & $\mathrm{b}$ \\
\hline
\end{tabular} \\
EWMA aggregator attributes
\end{tabular}

The same is for the EmailAlarm mixin, that has threshold, input and email attributes. It is posted to the AlarmonOverload sensor with:

\begin{tabular}{|l|l|}
\hline name & value \\
\hline threshold & "80" \\
email & "myself@example.com" \\
input & b \\
\hline
\end{tabular}

The operation, here split in a series of 5 POST operations, can be aggregated in a unique POST, using a suitable syntax defined for OCCI resources.

The user interface can be simplified by defining a template that embeds the whole structure, thus allowing the presence of a single button on an hypothetical graphic user interface. In the same spirit, the email alarm might be replaced with a WebSocket (Fette and Melnikov, 2011) connection driving a green/red light on the graphic user interface.

\section{Conclusions}

One conclusion of this work is about the OCCI ontology in itself. The fact that the core model can be used for a purpose distant from the original one (that was the description of IaaS provisions) confirms that it is a good standard, potentially stable in time. This is relevant as a guarantee of the return of an investment in compliance.

Monitoring has recently emerged as a relevant aspect of a cloud provisioning. This reflects on the interest for an extension of the OCCI core to express a monitoring infrastructure, which is the subject of this paper. Being based on the core model, it is potentially extensible to applications beyond the current horizon.

In this paper we have validated our ontology with respect to a number of providers that offer a cloud monitoring, showing that their services can be described using our model. By the time this paper will be published, an formal XML description of the model will be available, and we consider the implementation of a proof of concept prototype.

\section{REFERENCES}

Berners-Lee, T., Fielding, R., and Masinter, L. (2005). Uniform Resource Identifier (URI): Generic Syntax. RFC 3986 (INTERNET STANDARD). Updated by RFC 6874.

Bernstein, D. and Vij, D. (2010). Using semantic web ontology for intercloud director ies and exchanges. In International Conference on Internet Computing (ICOMP).

Ciuffoletti, A., Ferrari, T., Ghiselli, A., and Vistoli, C. (2003). Architecture of monitoring elements for the network element modeling in a grid infrastructure. In Proc. of Workskop on Computing in High Energy and Nuclear Physics, La Jolla (California).

Ciuffoletti, A. and Polychronakis, M. (2007). Architecture of a Network Monitoring Element, volume 4375 of Lecture Notes in Computer Science, chapter 2, pages 4-14. Springer.

Di Modica, G., Petralia, G., and Tomarchio, O. (2012). A semantic framework to support cloud markets in interoperable scenarios. In Utility and Cloud Computing (UCC), 2012 IEEE Fifth International Conference on, pages 211-214.

Edmons, A., Metsch, T., Papaspyrou, A., and Richardson, A. (2012). Toward an open cloud standard. IEEE Journals and Magazines, (4):1525 .

Fette, I. and Melnikov, A. (2011). The WebSocket Protocol. RFC 6455 (Proposed Standard).

Fielding, R. T. and Taylor, R. N. (2002). Principled design of the modern web architecture. ACM Trans. Internet Technol., 2(2):115-150.

Mell, P. and Grance, T. (2011). The NIST definition of cloud computing. Technical Report Special Publication 800-145, US Department of Commerce.

OGF (2011a). Open Cloud Computing Interface - Core. Open Grid Forum. Available from www.ogf.org. A revised version dated 2013 is available in the project repository.

OGF (2011b). Open Cloud Computing Interface - Infrastructure. Open Grid Forum. Available from www.ogf.org.

VMware (2007). Vmware high availability: Concepts, implementation and best practices. White paper.

Youseff, L., Butrico, M., and Da Silva, D. (2008). Toward a unified ontology of cloud computing. In Grid Computing Environments Workshop, 2008. $G C E^{\prime} 08$, pages $1-10$. 